\title{
Two-Dimensional Tunable and Temperature-Insensitive Lyot Filter for FM-to-AM Compensation
}

\author{
Mengqiu FAN ${ }^{1,2}$, Xiaocheng TIAN ${ }^{1}$, Dandan ZHOU ${ }^{1}$, Jiatian $\mathrm{WEI}^{3}$, Handing XIA ${ }^{1}$, \\ Hongwei $\mathrm{LV}^{3}$, Hao $\mathrm{ZHAO}^{3}$, Dangpeng $\mathrm{XU}^{1 *}$, and Wanguo $\mathrm{ZHENG}^{1}$ \\ ${ }^{1}$ Laser Fusion Research Center, China Academy of Engineering Physics, Mianyang 621900, China \\ ${ }^{2}$ Graduate School of China Academy of Engineering Physics, Beijing 100088, China \\ ${ }^{3}$ No. 34 Institute of China Electronics Technology Group Corporation, Guilin 541004, China \\ *Corresponding author: Dangpeng XUＥ-mail: xdpeng803@163.com
}

\begin{abstract}
Utilizing polarization maintaining photonic crystal fiber (PM-PCF) with the low temperature coefficient of birefringence, a two-dimensional tunable and temperature-insensitive Lyot filter aiming to compensate the frequency modulation to amplitude modulation (FM-to-AM) conversion in high power laser facility is demonstrated. The Jones matrix is applied to analyze the relationship between optical characteristics of the filter and physical parameters (including amplitude ratio, phase delay, and susceptibility of the birefringence to temperature) of the polarization optical field. Both the transmission peak wavelength and extinction ratio of the spectral transmission are able to be changed simultaneously, hence, it shows more efficient FM-to-AM compensation ability. Besides, the transmission peak shift is about $18 \mathrm{pm} /{ }^{\circ} \mathrm{C}$ with the PM-PCF configuration, which is about two orders of magnitude less than the normal polarization maintaining fiber (PMF) configuration. The demonstrated filter presents a practical application potential in large scale laser driven facility.
\end{abstract}

Keywords: Laser fusion; optical fiber devices; frequency modulation; birefringence; optical filters

Citation: Mengqiu FAN, Xiaocheng TIAN, Dandan ZHOU, Jiatian WEI, Handing XIA, Hongwei LV, et al., "Two-Dimensional Tunable and Temperature-Insensitive Lyot Filter for FM-to-AM Compensation," Photonic Sensors, 2021, 11(3): 325-333.

\section{Introduction}

Broadband spectrum in large scale laser facility which drives inertial confinement fusion (ICF) is essential due to the two reasons: suppressing of transverse stimulated Brillouin scattering (TSBS) and smoothing the speckle pattern illuminating the target [1-4]. Generally, a phase modulation is extensively adopted to broaden the spectrum of the single longitudinal mode seed source for matching the spectral requirement in ICF laser driver facility [5-7]. Ideally, the phase modulated pulse would maintain the original temporal shape when it propagates through the waveguides and optics with uniform spectral transmissions. However, the spectral transfer functions of the waveguides and optics are usually non-uniform due to various optical effects including the group velocity dispersion (GVD) [8], polarization mode dispersion (PMD) [9], gain-narrowing (GN), and weak etalon (WE) effect [10]. As a result, the frequency modulation (FM) is partly converted into amplitude modulation (AM). The FM-to-AM conversion can cause the intensity modulation in time domain and therefore degrades the power balance of the laser driver facility. Hence, the FM-to-AM conversion should be compensated

Received: 27 March 2020 / Revised: 24 May 2020

(C) The Author(s) 2020. This article is published with open access at Springerlink.com

DOI: $10.1007 / \mathrm{s} 13320-020-0591-4$

Article type: Regular 
to promote the performance of ICF laser driver facility. Most previous proposed FM-to-AM compensation technologies and devices are based on wave plates or crystals $[11,12]$. Although they have great compensation capability, they are lack of robustness and it is kind of hard to be all-fiber integrated due to waveplates or crystal materials. A compensation filter based on the polarization maintaining fiber (PMF) is presented in [13], while it needs a precision temperature controller to adjust the transmission peak wavelength, which makes the filter more complex and slow to respond. Besides, it still needs a half-wave plate to adjust the extinction ratio of the filter. In 2016, Zhi Qiao et al. [9] demonstrated an all polarization fiber (PZ) front-end system to suppress the FM-to-AM conversion, in which FM-to-AM $\alpha$ was only $4 \%$. It is a promising alternative for realizing the low FM-to-AM modulation. However, this kind of front-end system based on all PZ fiber is not an economical system at present due to the complex fabrication and high cost of all PZ fiber. Therefore, it is necessary to develop a more cost-efficient and robust technology to compensate FM-to-AM modulation.

In this paper, we demonstrate an all-fiber two-dimensional tunable Lyot filter utilizing polarization maintaining photonic crystal fiber (PM-PCF) adjusted by the polarization controller (PC) to compensate the FM-to-AM conversion. To the best of our knowledge, the relationship between the physical parameters of polarized optical field and the filter optical transmission features, including peak transmission wavelength, extinction ratio, and temperature sensitivity, is discussed comprehensively for the first time. Based on the theoretical analyses, we demonstrate that the transmission peak wavelength and extinction ratio of the proposed filter with the $\mathrm{PC}$ can be tuned synchronously. Moreover, by employing the PM-PCF, a stable spectral transmission of the demonstrated filter is realized, which is almost insulated to ambient temperature. In the compensation experiment, the FM-to-AM index $\alpha$ is effectively reduced from $17.8 \%$ to $1.74 \%$.

\section{Theoretical analysis}

In general, the Lyot birefringent filter basically consists of birefringent media such as the PMF, wave plates, and crystal between two polarizers [14-16]. Figure 1 shows the schematic setup of the proposed all-fibered Lyot filter. In our model, all the components are fiber-based devices. Without the loss of generality, the PMF is suitable for the birefringent fiber during the theoretical analysis process. Besides, the $\mathrm{PZ}$ fibers actually act as polarizers due tothe greater loss of the fast axis [17, 18]. However, the PZ fiber must exceed a certain length, otherwise it could not polarize the input light well. A fiber-based PC is inserted into the filter to manage the polarization ellipse. The input pulse is polarized along the $X$ direction when it propagates after a section of the PZ fiber which is equal to the first polarizer. Another section of the PZ fiber is arranged after the birefringent fiber and it plays as another polarizer. The birefringent fiber introduces phase difference. At last, $X$ and $Y$ components of the optical light interfere after the second PZ fiber and it results in a multi-wavelength optical spectral transmission. All the slow axes of the birefringent fiber and PZ fibers are aligned to the $X$ direction.

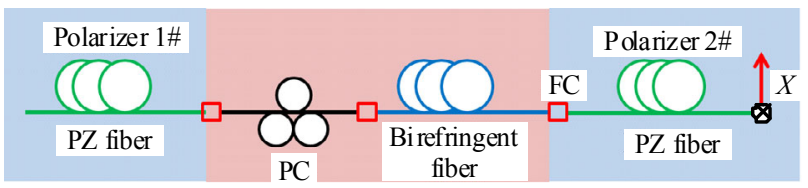

Fig. 1 Schematic setup (FC: fiber connector; $\otimes: Y$ direction).

To analyze the spectral transmission $H(\lambda)$ of the filter, we apply the Jones matrix method:

$$
\begin{gathered}
{\left[\begin{array}{c}
H(\lambda) \\
0
\end{array}\right]=} \\
\mathbf{P} \times \mathbf{R}\left(\phi_{3}\right) \times \mathbf{M}_{\mathrm{PMF}} \times \mathbf{R}\left(\phi_{2}\right) \times \mathbf{M}_{\mathrm{PC}} \times \mathbf{R}\left(\phi_{1}\right) \times\left[\begin{array}{l}
1 \\
0
\end{array}\right]
\end{gathered}
$$

where 


$$
\mathbf{R}\left(\phi_{j}\right)=\left[\begin{array}{cc}
\cos \left(\phi_{j}\right) & -\sin \left(\phi_{j}\right) \\
\sin \left(\phi_{j}\right) & \cos \left(\phi_{j}\right)
\end{array}\right]
$$

denotes the polarization rotation due to the polarization extinction ratio (PER) of the fiber connector. The rotation angle $\phi_{j}$ is determined by PER considering the definition $P E R_{j}=1 / \tan ^{2}\left(\phi_{j}\right)$. The typical value of PER is $23 \mathrm{~dB}$ from the vendor.

$$
\mathbf{M}_{\mathrm{PMF}}=\left[\begin{array}{cc}
1 & 0 \\
0 & \exp (-\mathrm{i} \varphi)
\end{array}\right] .
$$

$\mathbf{M}_{\mathrm{PMF}}$ represents the birefringence of the PMF, where $\varphi=2 \pi L \Delta n / \lambda$ is the phase delay accumulated between the fast and slow axes over the PMF. $\lambda$ is the operation wavelength and $\Delta n=n_{\text {slow }}-n_{\text {fast }}$ is the birefringence. $L$ is the fiber length of the birefringent fiber. $\mathbf{M}_{\mathrm{PC}}$ is the Jones matrix for the PC. It is feasible to treat the $\mathrm{PC}$ as a combination of a half-wave plate and a quarter-wave plate [19]:

$$
\begin{gathered}
\mathbf{M}_{\mathrm{PC}}=\mathbf{J}_{h} \times \mathbf{J}_{q}= \\
{\left[\begin{array}{cc}
\cos 2 \theta_{h} & -\sin 2 \theta_{h} \\
-\sin 2 \theta_{h} & -\cos 2 \theta_{h}
\end{array}\right] \times} \\
{\left[\begin{array}{cc}
-\cos ^{2} \theta_{q}+\mathrm{i} \sin ^{2} \theta_{q} & (\mathrm{i}-1) \cos \theta_{q} \sin \theta_{q} \\
(\mathrm{i}-1) \cos \theta_{q} \sin \theta_{q} & \mathrm{i} \cos ^{2} \theta_{q}+\sin ^{2} \theta_{q}
\end{array}\right]}
\end{gathered}
$$

where $\theta_{h}$ and $\theta_{q}$ represent the rotation angle of the half-wave plate and the quarter-wave plate relative to the $X$ axis, respectively. The last term $\mathbf{P}$ is the Jones matrix of the $\mathrm{PZ}$ fiber:

$$
\mathbf{P}=\left[\begin{array}{ll}
1 & 0 \\
0 & 0
\end{array}\right] .
$$

Then, the transmission $H(\lambda)$ variation with $\lambda$ is able to be calculated and plotted in Fig. 2. In our simulation, the PMF length is set as $3.8 \mathrm{~m}$ while the birefringence $\Delta n$ is assumed as $4.4 \times 10^{-4}$ referred in [12].

It is clear from Fig. 2 that the peak transmission wavelength spacing is $0.67 \mathrm{~nm}$ and both the peak transmission wavelength and extinction ratio of the spectral transmission could be tuned simultaneously. It is a progress comparing with our previous work [12]. Actually, the key reasons are amplitude ratio and phase delay of the two components along the $X$ and $Y$ axes, which are comprehensively dependent on the polarization azimuth angle with respect to the $X$ axis and ellipticity of polarization ellipse. Hence, it is of interest to analyze the above two effects.

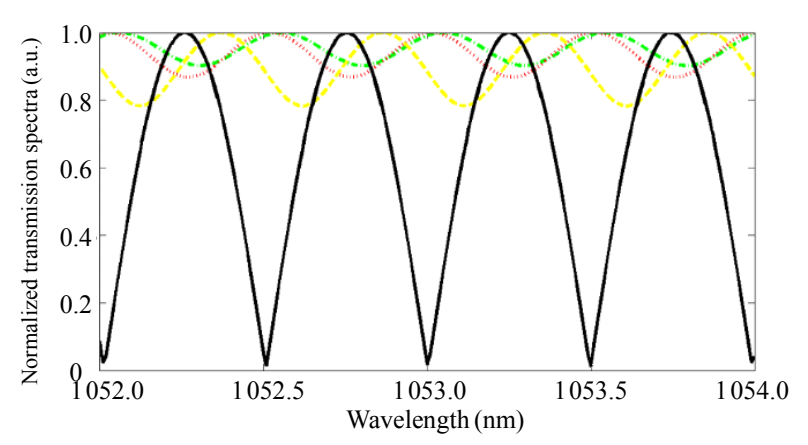

Fig. 2 Simulated spectral transmission $H(\lambda)$ (solid black curve: $\theta_{h}=\pi / 4$ and $\theta_{q}=0$; dashed yellow curve: $\theta_{h}=\pi / 6$ and $\theta_{q}=0$; dotted red curve: $\theta_{h}=0$ and $\theta_{q}=\pi / 4$; dotted dashed green curve: $\theta_{h}=0$ and $\left.\theta_{q}=\pi / 6\right)$.

Firstly, we consider the amplitude ratio of $X$ and $Y$ components. According to (4), $J_{h}$ does not include the imaginary part, so that the half-wave plate cannot arbitrarily change the phase difference (except $\pi$ ) but only changes the amplitude ratio of polarization components along $X$ and $Y$ axes. Therefore, it is interesting to investigate the amplitude ratio by rotating the half-wave plate. Besides, it is well-known that the polarization orientation will rotate $2 \theta_{h}$ when it passes after the half-wave plate [20]. We first fix the quarter-wave plate namely $\theta_{q}$ and rotate the half-wave plate from the $X$ axis for $\theta_{h}=15^{\circ}, 30^{\circ}, 45^{\circ}$, and $60^{\circ}$, respectively. The curves of the simulated spectral transmission $H(\lambda)$ are plotted in Fig. 3(a). Obviously, the extinction ratio of the spectral transmission is sensitive to $\theta_{h}$. However, the transmission peak wavelength is independent on $\theta_{h}$ when $\theta_{h}$ is less than $\pi / 4$. And the transmission peak wavelength shifts half period when $\theta_{h}$ exceeds $\pi / 4$. The reason is that the polarized light rotates across a quadrant and then the spectral transmission shifts to the opposite. According to the stated above, it is concluded that the amplitude ratio of two axes affects the depth of the spectral transmission. 
Secondly, we investigate the phase delay between $X$ and $Y$ components. The rotated quarter-wave plate has a useful property that it can be used to generate any desired orientation and ellipticity starting with an incident linearly polarized pulse [20]. However, it is unable to simply tune the phase delay and maintain the amplitude ratio. Therefore, we initialize the incident polarized pulse with phase delay $\delta$ as $\left[\begin{array}{c}1 \\ \exp (\mathrm{i} \delta)\end{array}\right]$ and remove PC (namely wave plates). Figure $3(\mathrm{~b})$ presents the simulated transmission $H(\lambda)$ case, in which only phase delay $\delta$ is adjusted from 0 to $\pi / 2$. Obviously, the phase delay $\delta$ only determines the peak transmission wavelength and the peak transmission wavelength shift is linearly dependent on $\delta$. Furthermore, when $\delta$ varies $\pi$, then, the rotation of optical field reverses and ellipticity remains unchanged. Consequently, the peak transmission wavelength shifts by a half cycle.

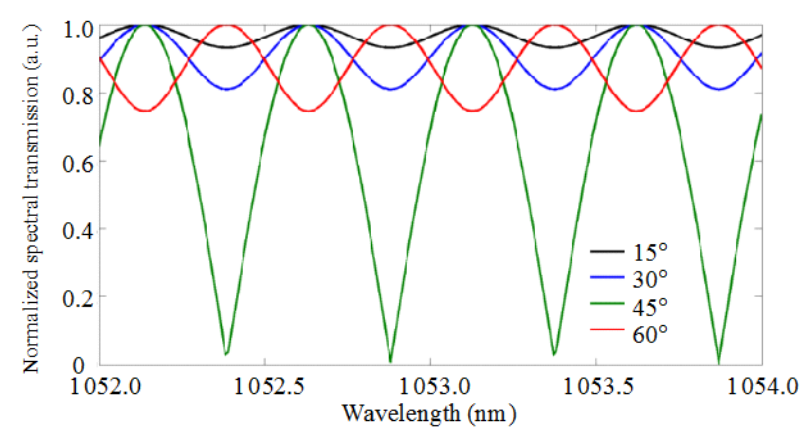

(a)

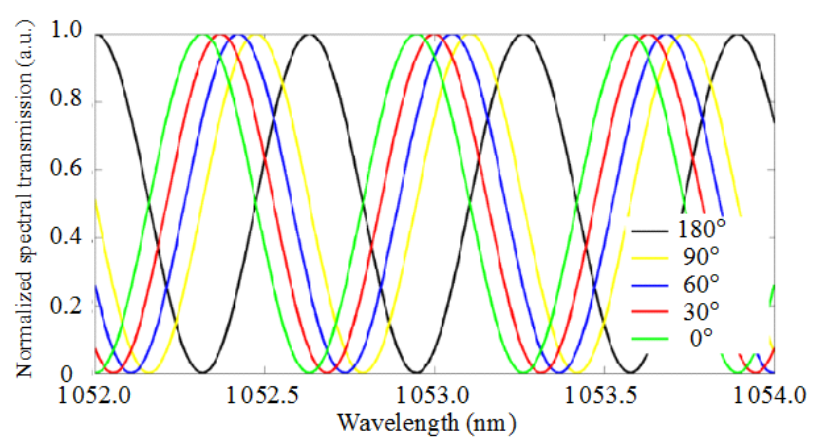

(b)

Fig. 3 Simulated spectral transmission $H(\lambda)$ : (a) $\theta_{q}$ is fixed while $\theta_{h}=15^{\circ}, 30^{\circ}, 45^{\circ}$, and $60^{\circ}$ and (b) PC is removed while phase delay $\delta$ is $0^{\circ}, 30^{\circ}, 60^{\circ}, 90^{\circ}$, and $180^{\circ}$.
Finally, the temperature sensitivity of the filter is investigated. The temperature variation mainly influences the phase difference $\varphi$ introduced by the birefringent fiber $[21,22]$ :

$$
\begin{aligned}
\frac{\mathrm{d} \varphi}{\mathrm{d} T} & =\frac{2 \pi}{\lambda}\left(\frac{\partial \Delta n}{\partial T} L+\frac{\partial L}{\partial T} \Delta n\right) \\
& =\frac{2 \pi L}{\lambda}\left(\frac{\partial \Delta n}{\partial T}+\frac{\partial L}{L \partial T} \Delta n\right) \\
& =\frac{2 \pi L}{\lambda}\left(\frac{\partial \Delta n}{\partial T}+\varepsilon \Delta n\right)
\end{aligned}
$$

where $T$ stands for temperature; $\partial \Delta n / \partial T$ represents the susceptibility of the birefringence to temperature; $\varepsilon$ is the thermal expansion coefficient although it is most often neglected in the common birefringent fiber. According to [21], the susceptibility of the birefringence to temperature of conventional PM980 fiber is of the order of $10^{-6} / \mathrm{K}-$ $10^{-7} / \mathrm{K}$. Here, the value of the susceptibility of the birefringence to temperature is set as $-9.5 \times 10^{-7}$ and other parameters are the same to Fig. 3. It should be noted that the sign of the susceptibility depends on the geometry of the fiber. The simulated spectral transmission with different temperatures based on the PM980 fiber is shown in Fig. 4. According to Fig. 4, the peak transmission wavelength shifts to the shorter wavelength $0.24 \mathrm{~nm}$ when temperature increases by $0.1^{\circ} \mathrm{C}$.

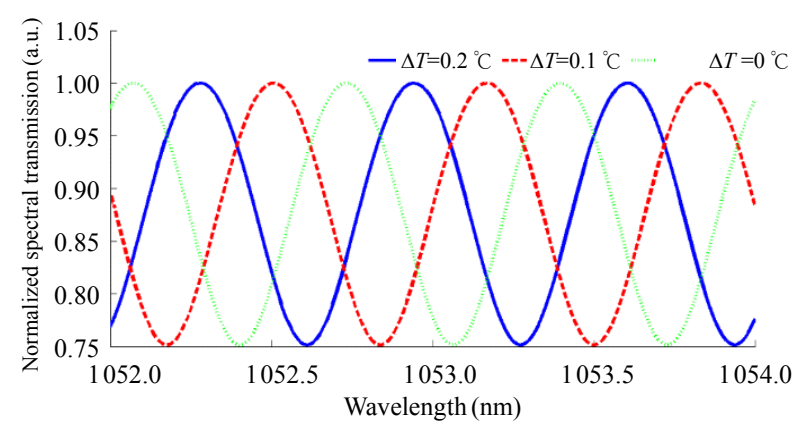

Fig. 4 Simulated spectral transmission with different temperatures based on the PM980 fiber.

\section{Spectral transmission experimental results}

To characterize the features especially the 
spectral transmission of our demonstrated filter, we design and conduct experiments to measure the optical spectral transmission. Figure 5 shows the measurement setup for the spectral transmission of the demonstrated two-dimensional tunable Lyot filter. As mentioned in Section 2, we empirically choose two sections of the $4 \mathrm{~m} \mathrm{PZ}$ fiber to act as the polarizer ensuring the polarizer effect. The birefringent fiber is first chosen to be a $3.8 \mathrm{~m}$ PM980 fiber which is bought from Nufern Company. The $\mathrm{PC}$ is a commercial manual three-paddle fiber-based device.

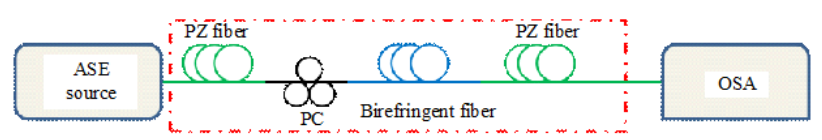

Fig. 5 Spectral transmission measurement setup.

Firstly, the output light of an amplified spontaneous emission (ASE) source propagates a section of the PZ fiber, and then its optical spectrum is measured by the optical spectrum analyzer (OSA) which owns $0.02 \mathrm{~nm}$ bandwidth resolution. Then, the optical spectrum is recorded again when ASE light propagates after our built filter. Next, the spectral transmission of the Lyot filter is the difference between the two measurements in the log scale. Finally, we rotate and adjust the PC with a full cycle and record other two spectral transmissions which are shown in Fig.6. In Fig. 6, the blue line has the maximum transmission (or the minimum loss of $1.6 \mathrm{~dB}$ ) while the extinction ratio is about $4.8 \mathrm{~dB}$. On the other hand, the green curve has the maximum extinction ratio of $17 \mathrm{~dB}$ while the loss is about $5 \mathrm{~dB}$. The wavelength spacing of the measured spectral transmission is $\sim 0.668 \mathrm{~nm}$ which is coordinated to our theoretical model. However, the maximum transmission is much lower than the simulation consequence. This can be interpreted as the significant polarization loss resulted from the single polarization operation principle of the $\mathrm{PZ}$ fiber [16, 17] and other fiber connection losses which are neglected in the simulation process. The most important thing is that the experimental result proves two-dimensional (peak transmission wavelength and extinction ratio) tunable features characterized by the PC.

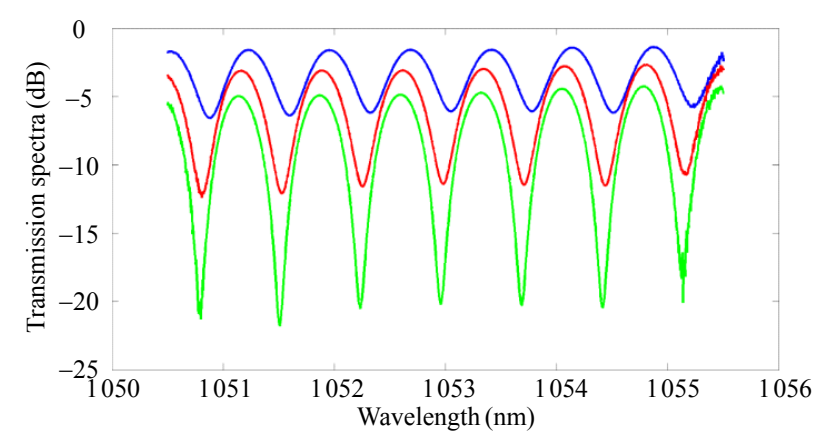

Fig. 6 Measured optical spectral transmissions based on the PM980 fiber with different PC states.

\section{Temperature-insensitive features}

The filter we demonstrate aims to stably compensate the FM-to-AM effect in the front-end system of the large-scale laser driver facility. Therefore, the filter must be insensitive to environment conditions, especially temperature. Obviously, the key point to realize the high stable transmission is the birefringence or the birefringent fiber. The PM-PCF is a special kind of the birefringent fiber which is made of only one material (and air holes), so that it has the identical thermal expansion coefficient, causing highly insensitive birefringence to temperature [23, 24]. In fact, the susceptibility of the birefringence to temperature of the PM-PCF also can be controlled during the design and manufacturing process of the PM-PCF according to $[21,22]$. In detail, the material and geometry of the PM-PCF determine the susceptibility of the birefringence to temperature. Therefore, it is possible to modify the parameters of the PM-PCF according to the simulation and experimental results. In general, the value of the susceptibility of the birefringence to temperature is two orders of magnitude larger than that of the PM980 fiber. For this reason, we use the PM-PCF as the birefringent fiber to improve the built filter.

Firstly, we simulate the temperature insensitive 
features of the PM-PCF configuration. The theoretical model follows the PM980 fiber configuration in Section 2, but the parameters are modified. The birefringence of the used PM-PCF is larger than $4 \times 10^{-4}$ at $1550 \mathrm{~nm}$ referring to the datasheet, which is bought from NKT company, PM-1550. Here, we modify the birefringence of the PM-PCF to $2.8 \times 10^{-4}$ at $1053 \mathrm{~nm}$ considering wavelength, and the fiber length is $4.4 \mathrm{~m}$. Besides, $\partial \Delta n / \partial T$ of the PM-PCF keeps the same order of magnitude comparing with that in [23] and is slightly modified as $-5 \times 10^{-9}$ according to our experimental results. Then, we calculate the spectral transmission with different temperatures based on the PM-PCF fiber. The simulation result is plotted in Fig. 7. According to Fig. 7, the wavelength spacing is about $0.9 \mathrm{~nm}$ and the peak transmission wavelength shifts $0.018 \mathrm{~nm} /{ }^{\circ} \mathrm{C}$, which is significantly different from the PM980 fiber configuration.

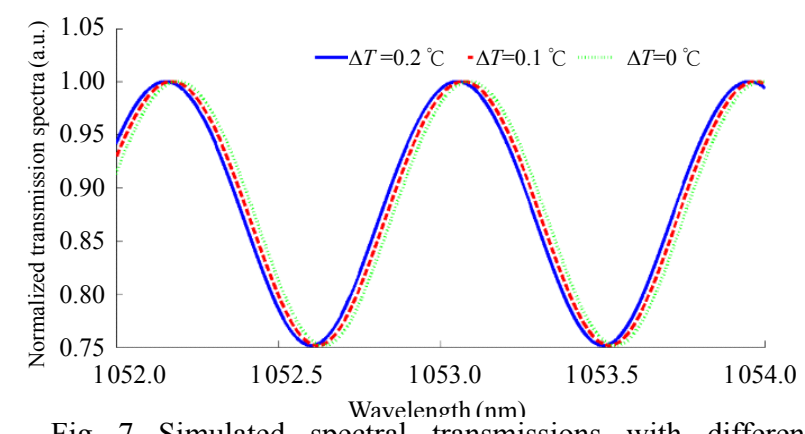

Fig. 7 Simulated spectral transmissions with different temperatures based on PM-PCF fiber.

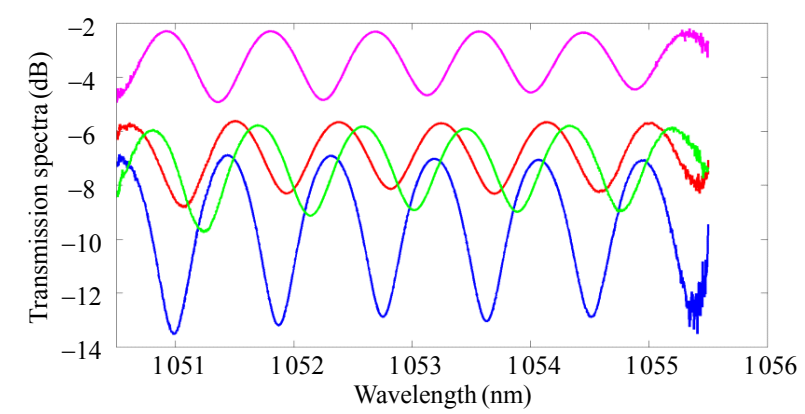

Fig. 8 Measured optical spectral transmission based on the PM-PCF fiber with different PC states.

Next, we experimentally build another twodimensional tunable filter based on the PM-PCF fiber. The length of the PM-PCF fiber is $4.4 \mathrm{~m}$ and other setups are the same with the PM980 fiber configuration. The spectral transmissions based on the PM-PCF are measured with different PC states following the mentioned steps in Section 3. The measurement results are shown in Fig. 8. The wavelength spacing is about $0.887 \mathrm{~nm}$, which is coordinated to the calculated result. Moreover, the PM-PCF configuration remains two-dimensional tunable features. Both the peak transmission wavelength and extinction ratio could also be tuned. Although the achievable minimum loss is about $2.3 \mathrm{~dB}$ which is a little higher than that of the PM980 configuration, it is acceptable considering higher loss resulted from the PCF and fiber fusion splice between the PCF and silica fiber.

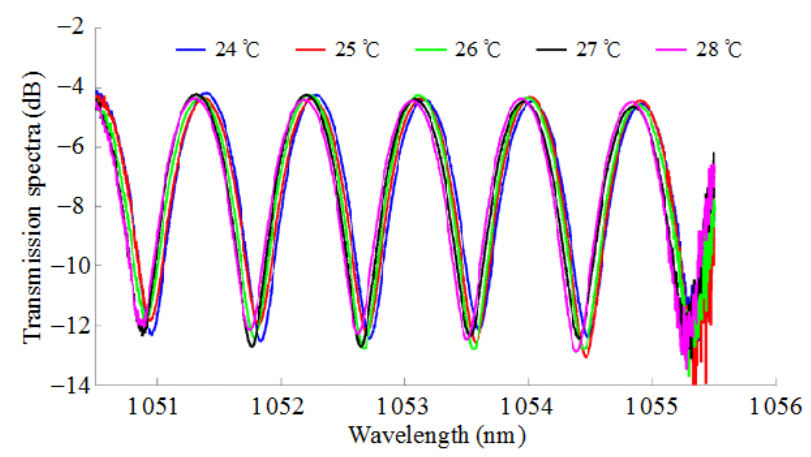

(a)

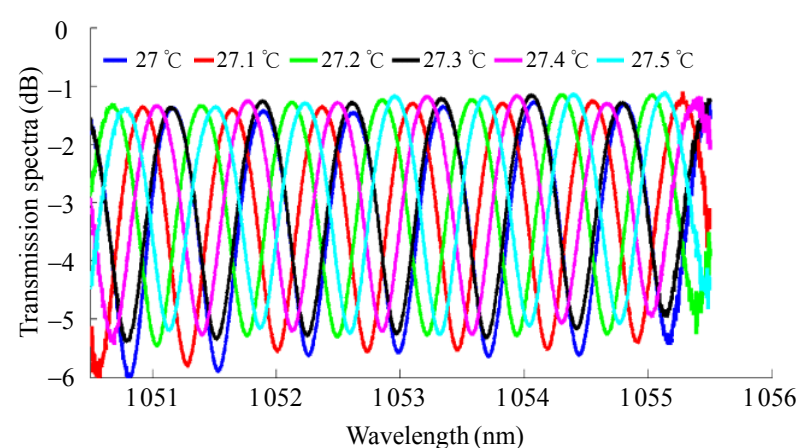

(b)

Fig. 9 Measured optical spectral transmission based on the (a) PM-PCF fiber and (b) PM980 fiber with different temperatures.

Then, we focus on the temperature insensitive features. In this experiment, we coil the birefringent fiber and stick it on a metal plate. Meanwhile, a thermo electric cooler (TEC) based temperature controller is applied and stuck on the opposite side of the metal plate to control the ambient temperature 
of the birefringent fiber. The temperature controller also contains the microprocessor, and detection and feedback circuit. Hence, the temperature controller has the high resolution ability of $0.001{ }^{\circ} \mathrm{C}$. Besides, the temperature controller is able to monitor the ambient temperature. It is possible for us to measure the spectral transmission with different temperatures. Figure 9(a) shows the measured optical spectral transmission based on the PM-PCF configuration with different temperatures from $24{ }^{\circ} \mathrm{C}$ to $28{ }^{\circ} \mathrm{C}$. When the temperature increases, the peak transmission wavelength shifts a little to the shorter wavelength. The wavelength shift of the peak transmission with temperature is about $0.018 \mathrm{~nm} /{ }^{\circ} \mathrm{C}$. In comparison, the spectral transmission curves based on the PM980 fiber are recorded and plotted in Fig. 9(b). When the temperature increases, the peak transmission wavelength shifts to the shorter wavelength, too. But the wavelength shift of the peak transmission with temperature is about $2.5 \mathrm{~nm} /{ }^{\circ} \mathrm{C}$, which is two orders of magnitude larger than that of the PM-PCF configuration.

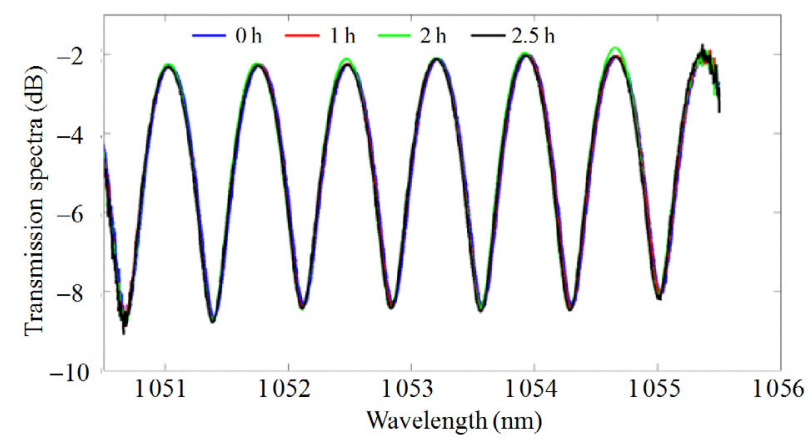

(a)

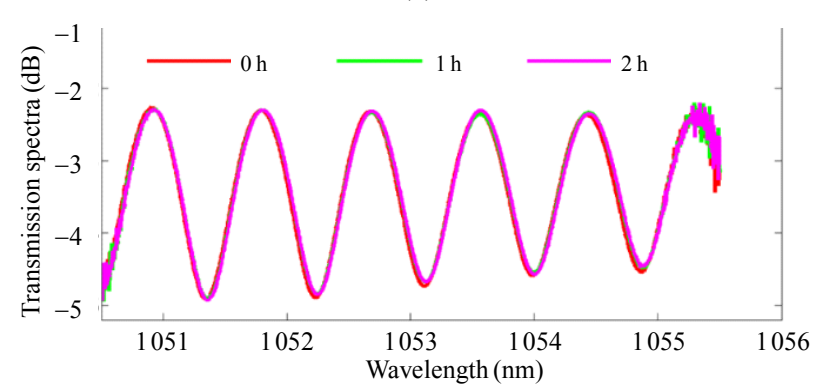

(b)

Fig. 10 Long-term optical spectral transmission based on the (a) PM980 fiber and (b) PM-PCF fiber.

Furthermore, we conduct the long-term spectral transmission output experiments and the results are shown in Fig. 10. Figure 10(a) shows the long-term optical spectral transmission based on the PM980 fiber. It should be pointed out that the temperature controller is running to obtain a stable long-term spectral transmission output based on the PM980 fiber. According to Fig. 10(a), the optical spectral transmission based on the PM980 and temperature controller rarely changes on the 2.5 -hour scale.

\section{FM-to-AM experimental results}

As we state above, the filter we demonstrate could tune the peak transmission wavelength and extinction ratio simultaneously. Moreover, it is insensitive to ambient temperature. Therefore, it is expected to compensate or pre-compensate the FM-to-AM effect of the fiber front-end system.

To verify the compensation ability, a conceptual experiment is demonstrated. The schematic diagram of the conceptual demonstrated experiment is shown in Fig. 11. A phase modulated squared pulse with $5 \mathrm{~ns}$ duration and certain energy is generated by the fiber front-end system. The pulse is phase modulated with $\sim 2.5 \mathrm{GHz}$ and it is expanded to $0.15 \mathrm{~nm}$ bandwidth. The signal pulse is seeded to the large mode area amplifier (LMA-AP) after the filter. The signal pulse is amplified from $n \mathrm{~J}$-level to $1 \mu \mathrm{J}$ in LMA-AP. Finally, we use a photo-detector with the $12 \mathrm{GHz}$ and $8 \mathrm{GHz}$ oscilloscope (OSC) to measure the temporal shape of the amplified pulse.

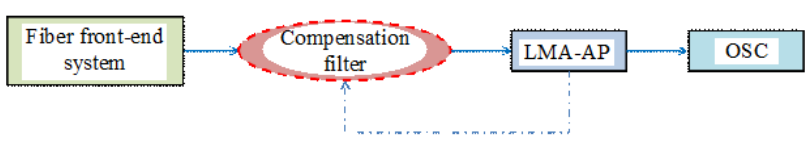

Fig. 11 Schematic diagram of FM-to-AM compensation.

Figure 12 shows the measured pulse shape. The blue solid curve is the original pulse shape outputs from the LMA-AP when the filter is standing by. The LMA-AP is based on the large mode area polarization fiber. So, the pulse accompanies a serious of temporal modulation due to PMD-induced FM-to-AM. Then, the compensation filter is running 
on and the red dotted curve presents the compensated pulse temporal shape. The modulation depth $\alpha$, which is defined in [3] is $17.8 \%$ while the modulation depth $\alpha$ of the compensated pulse, is reduced to $1.74 \%$ by adjusting the PC of the filter. It is verified as an effective way to manage the FM-to-AM modulation in the fiber front-end system.

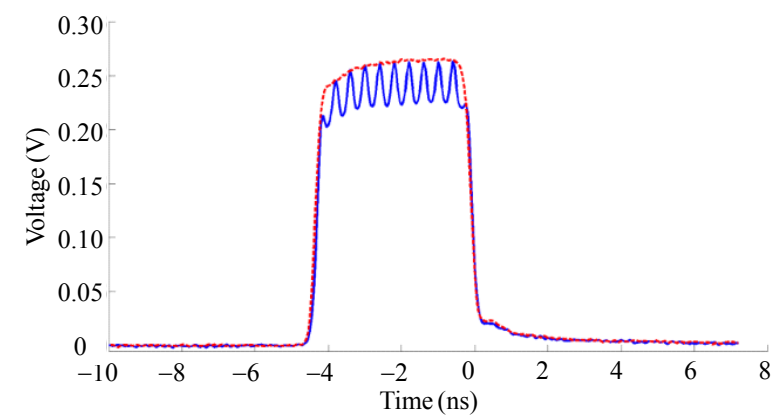

Fig. 12 Demonstrated FM-to-AM compensation experimental result.

\section{Conclusions and outlooks}

In this paper, we demonstrate a two-dimensional tunable (peak transmission wavelength and extinction ratio) and temperature insensitive birefringent filter. The Jones matrix method is developed to numerically analyze the characteristics of this filter. Especially, we reveal the relationship between the spectral transmission and physical parameters of the optical field. The peak transmission wavelength depends on the amplitude ratio of polarization components along $X$ and $Y$ axes. The extinction ratio relies on the phase difference of polarization components along $X$ and $Y$ axes. The temperature insensitive features are also theoretically and experimentally investigated. The peak transmission wavelength shifts $0.018 \mathrm{~nm} /{ }^{\circ} \mathrm{C}$ with the PM-PCF configuration, which is two orders of magnitude less than that of the conventional PM980 fiber. Finally, the FM-to-AM compensation experiment in the front-end system is demonstrated. The FM-to-AM conversion index $\alpha$ is reduced from $17.8 \%$ to $1.74 \%$.

According to the results, the demonstrated two-dimensional tunable and temperature- insensitive Lyot filter is able to effectively compensate the FM-to-AM. In fact, it is also believed that the demonstrated fibered Lyot filter is the practical choice to compensate the FM-to-AM in future when electric polarization control and feedback technique is adopted. Fortunately, the polarization stabilizer based on polarization control and feedback technique has been completely verified in laser facility [4]. Therefore, the two-dimensional tunable and temperatureinsensitive fibered Lyot filter shows the great robust and economical compared with other compensation method including all PZ fiber and temperature controlled Lyot filter.

\section{Acknowledgment}

This work was supported by the Science and Technology Major Project of Guangxi (Grant No. AA18118032) and Laser Fusion Research Center Funds for Young Talents (Grant No. RCFCZ3-2019-7).

Open Access This article is distributed under the terms of the Creative Commons Attribution 4.0 International License (http://creativecommons.org/licenses/by/4.0/), which permits unrestricted use, distribution, and reproduction in any medium, provided you give appropriate credit to the original author(s) and the source, provide a link to the Creative Commons license, and indicate if changes were made.

\section{References}

[1] J. Rothenberg, D. Browning, and R. Wilcox, "Issue of FM to AM conversion on the national ignition facility," in Third International Conference on Solid State Lasers for Application to Inertial Confinement Fusion, Monterey, CA, United States, 1999, pp. 51-61.

[2] J. D. Lindl, P. Amendt, and R. L. Berger, "The physics basis for ignition using indirect-drive targets on the national ignition facility," Physics of Plasmas, 2004, 11(2): 339-491.

[3] S. Hocquet, D. Penninckx, E. Bordenave, C. Gouédard, and Y. Jaouën, "FM-to-AM conversion in high-power lasers," Applied Optics, 2008, 47(18): 3338-3349.

[4] W. G. Zheng, X. F. Wei, Q. H. Zhu, F. Jing, D. X. 
Hu, X. D. Yuan, et al., "Laser performance upgrade for precise ICF experiment in SG-III laser facility," Matter and Radiation at Extremes, 2017, 2(5): 243-255.

[5] C. Dorrer, A. V. Okishev, R. G. Roides, R. Cuffney, W. Bittle, and J. D. Zuegel, "Fiber front end for an OMEGA EP demonstration of beam-smoothing techniques for NIF polar-drive ignition," in Conference on Lasers and Electro-Optics 2012, San Jose, California, USA, May, 2012, pp. CTu3M.2.

[6] J. F. Gleyze, J. Hares, S. Vidal, N. Beck, J. Dubertrand, and A. Perrin, "Recent advances in the front-end sources of the LMJ fusion laser," in High Power Lasers for Fusion Research, San Francisco, California, USA, February, 2011, pp. 79160I.

[7] D. P. Xu, X. C. Tian, D. D. Zhou, Z. Y. Zong, M. Q. Fan, R. Zhang, et al., "Temporal pulse precisely sculpted millijoule-level fiber laser injection system for high-power laser driver," Applied Optics, 2017, 56(10): 2661-2666.

[8] R. Li, W. Fan, Y. E. Jiang, Z. Qiao, P. Zhang, and Z. Q. Lin, "Tunable compensation of GVD-induced FM-AM conversion in the front end of high-power lasers," Applied Optics, 2017, 56(4): 993-998.

[9] Z. Qiao, X. C. Wang, W. Fan, X. C. Li, Y. E. Jiang, R. Li, et al., "Suppression of FM-to-AM modulation by polarizing fiber front end for high-power lasers," Applied Optics, 2016, 55(29): 8352-8358.

[10] D. P. Xu, J. J. Wang, M. Z. Li, H. Lin, R. Zhang, Y. Deng, et al., "Weak etalon effect in wave plates can introduce significant FM-to-AM modulations in complex laser systems," Optics Express, 2010, 18(7): 6621-6627.

[11] Z. Y. Chen, Y. E. Jiang, J. F. Wang, W. Fan, and X. C. Li, "Compensation system for FM-to-AM effects in high-power laser system," in Applied Optics and Photonics China, AOPC 2015, Beijing, China, 2015, pp. 9671.

[12] D. P. Xu, Z. H. Huang, J. J. Wang, M. Z. Li, H. H. Lin, R. Zhang, et al., "A fiber-based polarization-rotation filter utilized to suppress the FM-to-AM effect in a large-scale laser facility," Journal of Optics, 2013, 15(8): 085702: 1-4.

[13] S. Vidal, J. Luce, and D. Penninckx, "Experimental demonstration of linear precompensation of a nonlinear transfer function due to second-harmonic generation," Optics Letters, 2011, 36(1): 88-90.

[14] M. X. Wang, S. N. Fu, P. Shum, N. Q. Ngo, J. Wu, and J. T. Lin, "A tunable Lyot birefringent filter with variable channel spacing and wavelength using nonlinear polarization rotation in an SOA", IEEE Photonics Technology Letters, 2008, 20(18): 1527-1529.

[15] D. Penninckx, N. Beck, J. F. Gleyze, and L. Videau, "Signal propagation over polarization-maintaining fibers: problem and solutions," Journal of Lightwave Technology, 2006, 24(11): 4197-4207.

[16] S. Liu, F. P. Yan, F. Ting, L. N. Zhang, Z. Y. Bai, W. G. Han, et al., "Multi-wavelength thulium-doped fiber laser using a fiber-based Lyot filter," IEEE Photonics Technology Letters, 2016, 28(8): 864-867.

[17] J. R. Simpson, R. H. Stolen, F. M. Sears, W. Pleibel, J. B. Macchesney, and R. E. Howard, "A single-polarization fiber," Journal of Lightwave Technology, 1983, 1(2): 370-374.

[18] D. A. Nolan, G. E. Berkey, M. J. Li, X. Chen, W. A. Wood, and L. A. Zenteno, "Single-polarization fiber with a high extinction ratio," Optics Letters, 2004, 29(16): 1855-1857.

[19] H. Sunnerud, C. J. Xie, M. Karlsson, R. Samuelsson, and P. A. Andrekson, "A comparison between different PMD compensation techniques," Journal of Lightwave Technology, 2002, 20(3): 368.

[20] D. H. Goldstein, Polarized light. Florida: CRC Press, 2011.

[21] T. Martynkien, M. Szpulak, and W. Urbanczyk, "Modeling and measurement of temperature sensitivity in birefringent photonic crystal holey fibers," Applied Optics, 2005, 44(36): 7780-7788.

[22] L. Lu, Y. H. Yang, and H. Y. Li, "Study of polarization-maintaining photonic crystal fibers with zero birefringent temperature sensitive coefficient," Acta Optica Sinica, 2015, 35(10): 1006006.

[23] C. L. Zhao, X. F. Yang, C. Lu, W. Jin, and M. S. Demokan, "Temperature-insensitive interferometer using a highly birefringent photonic crystal fiber loop mirror," IEEE Photonics Technology Letters, 2004, 16(11): 2535-2537.

[24] G. W. Fu, Y. P. Li, X. H. Fu, W. Jin, and W. H. Bi, "Temperature insensitive curvature sensor based on cascading photonic crystal fiber," Optical Fiber Technology, 2018, 41: 64-68. 